\title{
A Rare Occurrence of Scalp Metastasis in Hepatocellular Carcinoma: Case Report
}

\author{
Samreen Javed Chaudry ${ }^{1} \quad$ Fajar Rafi Ranjha ${ }^{1} \quad$ Sumera Butt ${ }^{1} \quad$ Samaha Nawaz ${ }^{1}$ \\ 1Department of Clinical \& Radiation Oncology, Shaukat Khanum \\ Memorial Cancer Hospital, Lahore, Pakistan

\begin{abstract}
Address for correspondence Samreen Javed Chaudry, MBBS, FCPS, Department of Clinical \& Radiation Oncology, Shaukat Khanum Memorial Cancer Hospital, 7 A Block R3, Johar Town, Lahore, Pakistan (e-mail: samreenjchaudry@gmail.com).
\end{abstract}

Ind J Med Paediatr Oncol 2021;42:390-393.

\begin{abstract}
Keywords

- hepatocellular

carcinoma

- meningioma

- scalp metastasis
\end{abstract}

\section{Introduction}

Among primary carcinoma of the liver, hepatocellular is the most common. Regional lymph nodes and lungs and less commonly bones are the frequent sites of metastasis. ${ }^{1}$ Upfront scalp metastasis occurs in only 0.47 to $1.6 \%$ of all hepatocellular carcinoma (HCC) with only a handful of reports in the literature. ${ }^{2}$ Only seven similar cases have been published so far. This case report emphasizes that metastatic scalp lesion should be considered a differential diagnosis in HCC regardless of liver symptoms. In this case report, we present a rare case of HCC with scalp metastasis misdiagnosed as meningioma initially. The patient and his family provided their consent for publication.

\section{Case Report}

A middle-aged Asian male patient had a lump on his scalp for the last 14 months; it was painless on presentation, with recent rapid enlargement for which he underwent craniotomy and excision in a local facility under the tentative diagnosis of brain tumor. There was no associated history of headache, vomiting, or any neurological deficit. ECOG (Eastern Cooperative Oncology Group) performance status was 0 . The patient's medical history was significant for previously treated hepatitis $C$ virus-related hepatitis and pulmonary tuberculosis (TB).

Physical examination revealed a lump on the left parietal region with an oozing wound $\sim 8.5 \mathrm{~cm}$ in diameter. Upon examination, it was red and nonpulsatile with a negative cough impulse. General and systemic examinations were unremarkable with no signs indicating chronic liver or brain pathology. The histopathology report of the excised mass at an outside facility was consistent with meningioma Grade III.

He was referred to our hospital for postoperative radiotherapy to the scalp. The case was discussed in our multidisciplinary team and histopathology slides were reviewed. Brain imaging (magnetic resonance imaging [MRI]) revealed a left-sided extra-axial mass (-Figs. $\mathbf{1}$ and $\mathbf{2}$ ).
Dol https://doi.org/

10.1055/s-0041-1736679

ISSN 0971-5851
(C) 2021. Indian Society of Medical and Paediatric Oncology

This is an open access article published by Thieme under the terms of the Creative Commons Attribution-NonDerivative-NonCommercial-License, permitting copying and reproduction so long as the original work is given appropriate credit. Contents may not be used for commercial purposes, or adapted, remixed, transformed or built upon. (https://creativecommons.org/licenses/by-nc-nd/4.0/).

Thieme Medical and Scientific Publishers Private Ltd. A-12, Second Floor, Sector -2, NOIDA -201301, India 


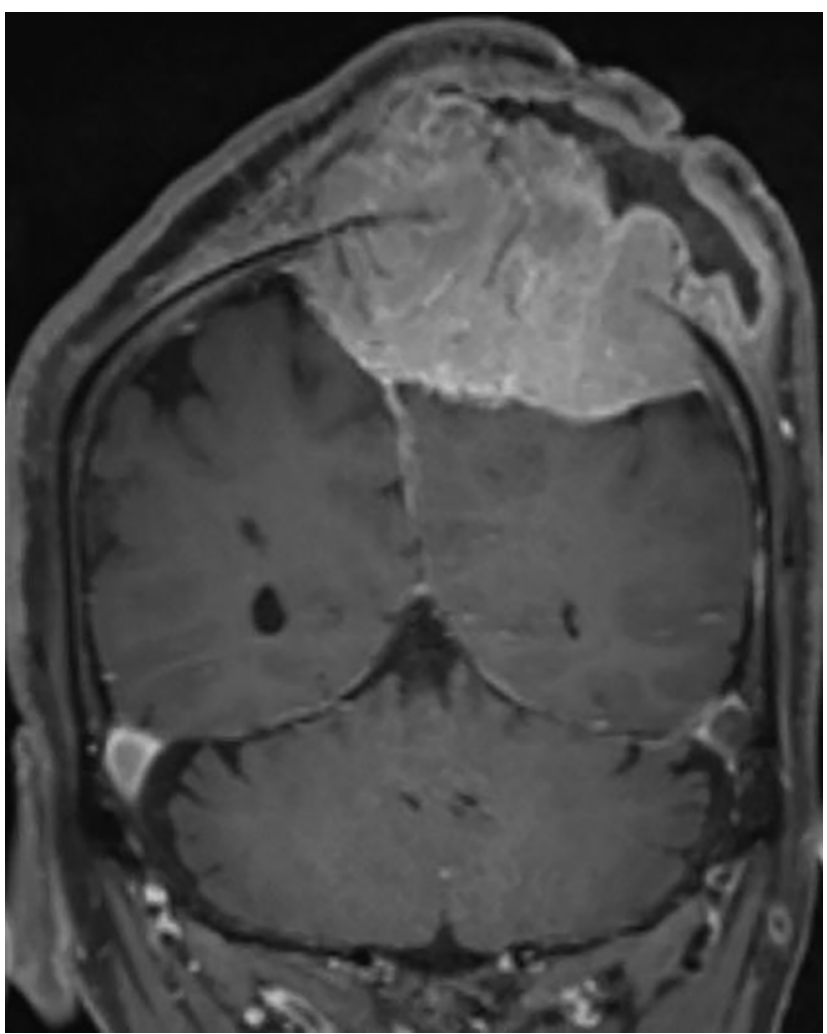

Fig. 1 Postgadolinium-enhanced coronal T1-weighted image showing enhancing large extra-axial dural-based mass in the vertex and left parietal region, involving the overlying scalp soft tissue. There was no intra-axial parenchymal extension.

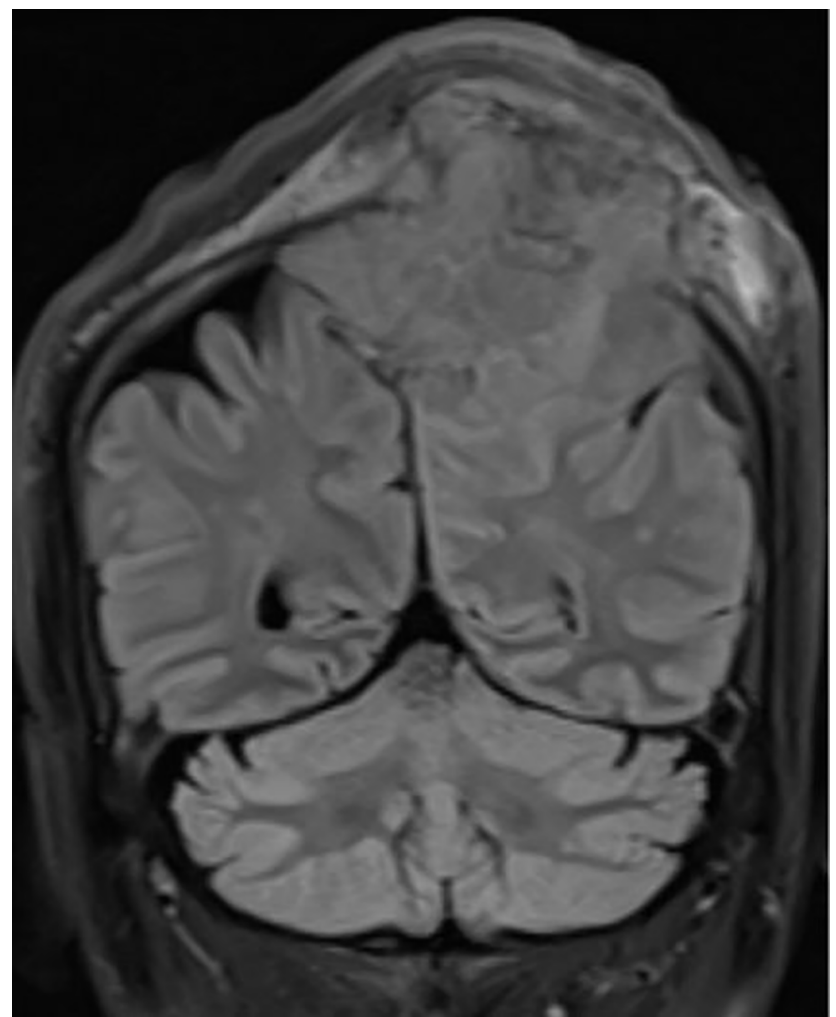

Fig. 2 Fluid-attenuated inversion recovery magnetic resonance imaging showing enhancing large extra-axial dural-based mass in the vertex and left parietal region, involving the overlying scalp soft tissue. There was no intra-axial parenchymal extension.

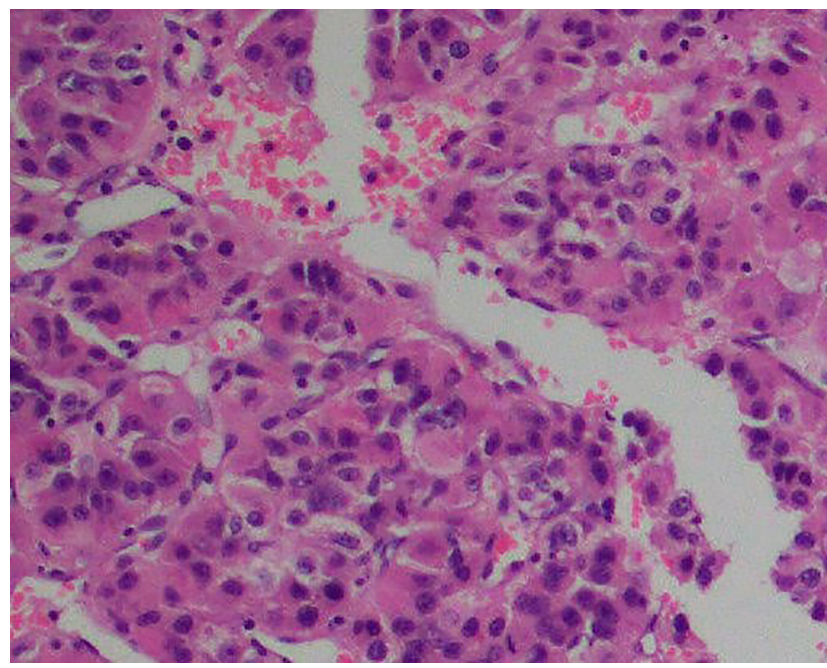

Fig. 3 Positive cytokeratin staining (x20).

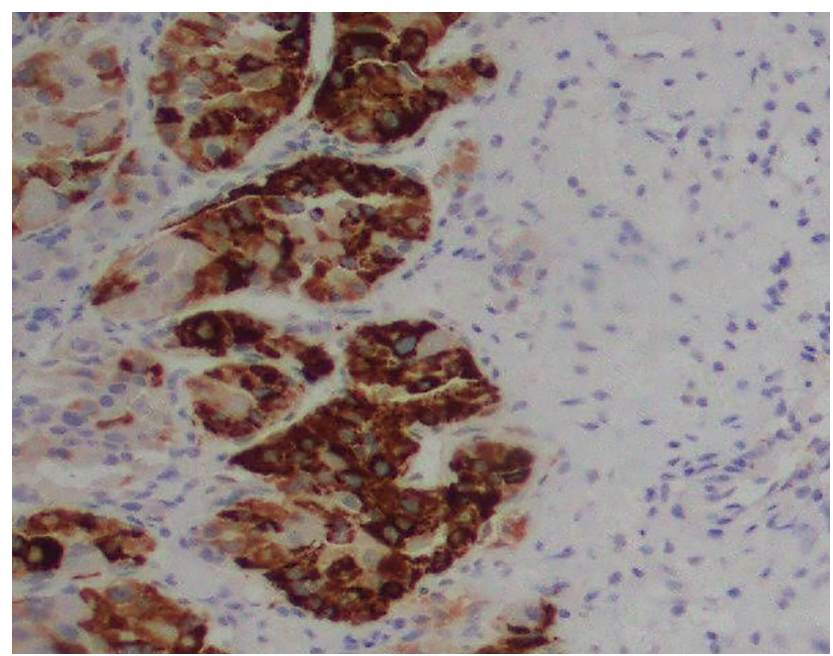

Fig. 4 Positive HepPar-1 staining.

Histological findings favored HCC. Immunohistochemical stains HepPar-1 and cytokeratin confirmed hepatic origin ( - Figs. 3 and 4 ).

His initial workup included a liver profile, a triphasic computed tomography (CT) abdomen, renal profile, complete blood count, and electrolytes, all of which were normal. His $\alpha$-fetoprotein levels were $12.7 \mathrm{IU} / \mathrm{mL}$. CT chest and abdomen showed a lesion in the one lung, which was consistent with $\mathrm{TB}$, and a lesion in the lumbar vertebrae. MRI spine with contrast confirmed the lesion as metastatic ( - Fig. $\mathbf{5}$ ).

Multiparametric MRI liver was performed that showed multiple lesions in the liver, hence supporting the pathological diagnosis of metastatic HCC to the scalp and lumbar vertebrae (-Fig. 6).

The patient was hence offered palliative external-beam radiation therapy. A palliative radiation dose of 2000 cGy in five fractions with $400 \mathrm{cGy}$ per fraction was prescribed and delivered for 5 days a week. Keeping in view the Child's Class A a targeted agent sorafenib $400 \mathrm{mg}$ twice daily was started. On 1-year follow-up, a marked reduction in the size of the scalp lesion was observed with almost no effect on the quality of life. 


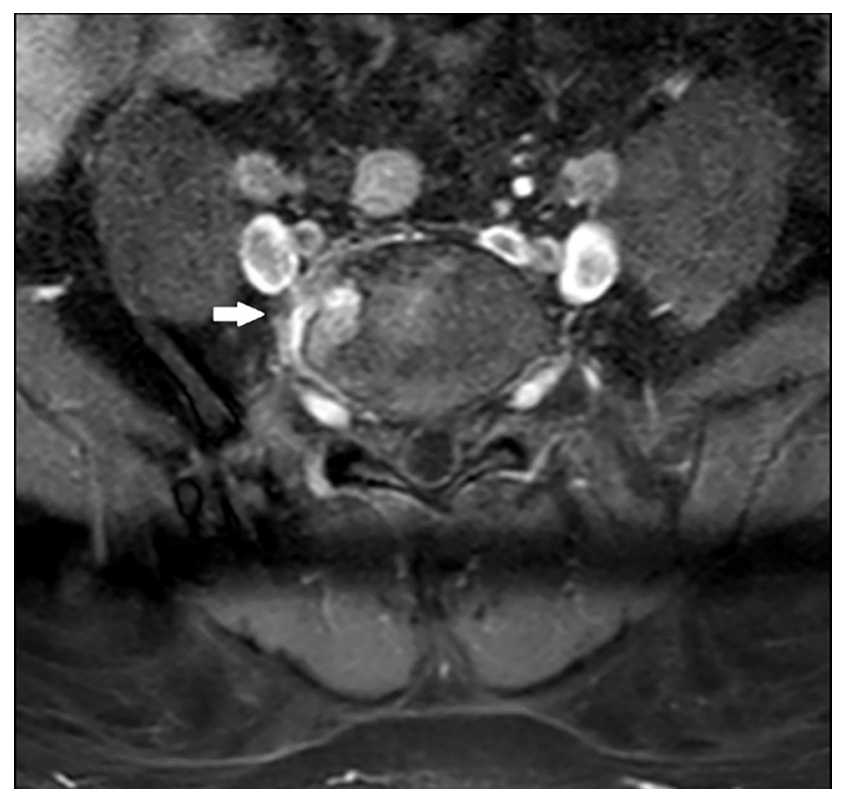

Fig. 5 Postgadolinium-enhanced magnetic resonance imaging axial slice through the $\mathrm{L} 5$ vertebra showing an enhancing lesion in the body of $\mathrm{L} 5$ vertebra anteriorly extending into the right pedicle. The sleeve of the soft tissue presents anteriorly in the pre- and paravertebral space without psoas infiltration. The sleeve of the soft tissue presents anteriorly in the pre- and paravertebral space without psoas infiltration (indicated by arrow).
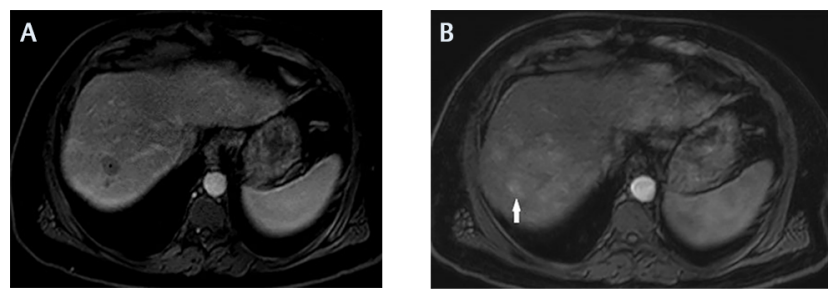

Fig. 6 Postgadolinium-enhanced axial magnetic resonance imaging of the abdomen showing a nodular lesion in the hepatic segment $7 / 8$ with patchy arterial enhancement (a) and washout on delayed sequence (arrow). (b) Postgadolinium-enhanced axial magnetic resonance imaging of the abdomen showing washout on delayed sequence (arrow).

\section{Discussion}

HCC is one of the most common but lethal causes of cancer-related deaths. HCC prevalence is strongly associated with hepatitis $\mathrm{B}$ and $\mathrm{C}$, accounting for high rates of occurrence in the Eastern world including Asia and sub-Saharan Africa. ${ }^{3,4}$ In the USA and many other European countries, there has been an uptrend toward contracting hepatitis $C$ infection, leading to an increase in the development of HCC in the west side of the world. ${ }^{5,6}$ Among other risk factors, alcohol, aflatoxins, hemochromatosis, nonalcoholic steatohepatitis, and metabolic syndromes are also associated with HCC. With the introduction of transcatheter arterial chemoembolization, radiofrequency ablation, and other advances in the surgical and medical management of HCC, the life expectancy has considerably improved, unveiling the incidence of distant metastasis in these patients. ${ }^{7.8}$
The number of deaths from HCC is considerably higher than its incidence owing to multiple factors including comorbidities, poor function of the rest of the liver, and misdiagnosis of secondary malignancies as the primary disease. Due to the poor long-term survival of HCC, $13 \%$ of incidences of distant metastasis have been documented at 5 years. ${ }^{9,10}$ Extrahepatic spread in HCC is most commonly seen in the lungs (47\%) and lymph nodes (45\%) and rarely in the skeletal system (16\%). ${ }^{1,10}$

Bone vertebrae and ribs are frequent sites of metastasis; skull lesions are extremely rare with a handful of cases reported worldwide until date. Upfront scalp metastasis occurs in only 0.47 to $1.6 \%$ of all HCCs. ${ }^{2}$ Due to the rarity of the disease, the natural history has not been fully understood, so the management of these in terms of improved survival outcomes remains a challenge.

A thorough review of the literature suggests that most of these patients present clinically for the first time with soft cystic subcutaneous scalp mass without signs of neurological deficits. ${ }^{11,12}$ Others mentioned slight tingling over the lesion along with headaches and hemiparesis. ${ }^{7,8,13}$

Two-thirds of these patients show a strong association with hepatitis B or C. The diagnosis of HCC was made on the histopathological specimen with immunohistochemical staining positive for HepPar- 1 and $\alpha$-fetoprotein. The workup for a secondary tumor has led to the diagnosis of primary tumor in almost all these cases, with the liver being studded with single or multiple lesions on CT scans.

\section{Conclusion}

The treatment modality standards have not been established so far; however, the intent to keep the approach palliative remains undoubted. Hence, radiotherapy seems to be a reasonable treatment option for local disease control.

\section{Patient Consent}

The authors certify that they have obtained all appropriate patient consent forms. In the form, the patient has given her consent for images and other clinical information to be reported in the journal. The patient understands that name and initials will not be published and due efforts will be made to conceal the identity, but anonymity cannot be guaranteed

\section{Funding}

None.

\section{Conflicts of Interest}

None declared.

\section{References}

1 Fukutomi $M$, Yokota $M$, Chuman $H$, et al. Increased incidence of bone metastases in hepatocellular carcinoma. Eur J Gastroenterol Hepatol 2001;13(9):1083-1088

2 Kuhlman JE, Fishman EK, Leichner PK, Magid D, Order SE, Siegelman SS. Skeletal metastases from hepatoma: frequency, distribution, and radiographic features. Radiology 1986;160(1):175-178

3 Choi HJ, Cho BC, Sohn JH, et al. Brain metastases from hepatocellular carcinoma: prognostic factors and outcome: brain metastasis from HCC. J Neurooncol 2009;91(3):307-313 
4 Jiang XB, Ke C, Zhang GH, et al. Brain metastases from hepatocellular carcinoma: clinical features and prognostic factors. BMC Cancer 2012;12:49

5 Rahbari NN, Mehrabi A, Mollberg NM, et al. Hepatocellular carcinoma: current management and perspectives for the future. Ann Surg 2011;253(3):453-469

6 Seinfeld J, Wagner AS, Kleinschmidt-DeMasters BK. Brain metastases from hepatocellular carcinoma in US patients. J Neurooncol 2006;76(1):93-98

7 Yoshida D, Chen MN, Awaya S, Nakazawa S. Cranial metastasis of hepatocellular carcinoma in a female-case report. Neurol Med Chir (Tokyo 1993;33(12):839-844

8 Hsieh CT, Sun JM, Tsai WC, Tsai TH, Chiang YH, Liu MY. Skull metastasis from hepatocellular carcinoma. Acta Neurochir (Wien 2007;149(2):185-190
9 Kanda M, Tateishi R, Yoshida H, et al. Extrahepatic metastasis of hepatocellular carcinoma: incidence and risk factors. Liver Int 2008;28(9):1256-1263

10 Uchino K, Tateishi R, Shiina S, et al. Hepatocellular carcinoma with extrahepatic metastasis: clinical features and prognostic factors. Cancer 2011;117(19):4475-4483

11 Subasinghe D, Keppetiyagama CT, Sudasinghe H, Wadanamby S, Perera N, Sivaganesh S. Solitary scalp metastasis - a rare presentation of hepatocellular carcinoma. Ann Surg Innov Res 2015;9:4

12 Shim YS, Ahn JY, Cho JH, Lee KS. Solitary skull metastasis as initial manifestation of hepatocellular carcinoma. World J Surg Oncol 2008;6:66

13 McIver JI, Scheithauer BW, Rydberg CH, Atkinson JL. Metastatic hepatocellular carcinoma presenting as epidural hematoma: case report. Neurosurgery 2001;49(2):447-449 